\section{SURVIVAL OF THE FITTEST}

Our not-too-distant past is decorated with artefacts: strategies that became popular for perfectly tenable reasons, had a Warholian 15 min of (perfectly justified) fame and then, as new perspectives developed were consigned to the museums of (spectacles rose- tinted) folklore or (spectacles replaced by blinkers) closed chapters 'we'd rather not discuss'. There is also, though, another, third, group: those practices that have evolved and improved as a result of a recognition of limitations and evolution. In geological terms at least, it wasn't that long (mid 1980s) since I was a medical student when the roll call of popular interventions included the mist tent in croup. This involved creating a fog in which 1 year-old children became not only detached from their parents but distressed by their treatment in a polythene tent draped over their cot (figure 1).

Other practices in use at that time or shortly after included the use of the lateral neck X-ray in children with suspected epiglottitis, lumbar puncture in all children with a first febrile seizure under the age of 18 months (even if they were happily running around the ward and near impossible to catch) and routine intubation and saline lavage for all neonates with meconium staining to 'cover the risk of aspiration' - great for practice, likely of very limited benefit in terms of outcomes.

We do our best, live, learn and adapt

This month's examples are from group 3: excellent in principle, have evolved, and, as a result, are here to stay in one form or another

\section{PAEDIATRIC EMERGENCY MEDICINE The rise, 'saturation' by and rethink of early warning scores}

After a honeymoon period noticeable for its uncritical reception and (in many cases) lack of objective assessment, paediatric early warning scores (PEWS) proliferated exponentially to the point

Department of Women's and Children's Health, International Maternal and Child Health (IMCH), Uppsala University, Uppsala, Sweden; Department of Paediatrics, Länssjukhuset Gävle-Sandviken, Gävle, Sweden; Department of Child Health, Aga Khan University, Karachi, Pakistan

Correspondence to Dr Nick Brown, Department of Women's and Children's Health, International Maternal and Child Health (IMCH), Uppsala University, Uppsala 752 37, Sweden; nickjwbrown@gmail.com

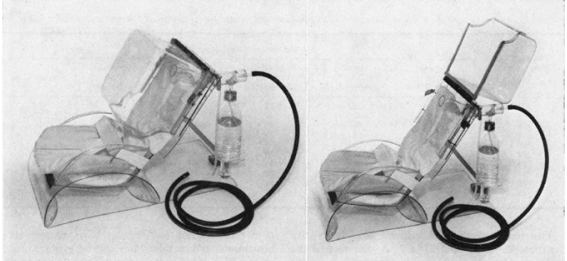

Figure 1 The mist tent for croup. Gomez: Archives 1968.

of submersion over a short period. There was a (although well-intentioned) degree of naivete in this unbounded parameterdriven enthusiasm. The proliferation, of course, for all the excellent intentions, was part of the problem: there were simply too many in use and it was impossible to familiarise with more than a small proportion of them all. That, of course, was part of the problem: we know now that human factors (inconsistency and interobserver variability) and insensitivities in the tools themselves (decompensation is often more subtle than measurable physiological deterioration) contribute to their imperfections. The largest of the red flags came in the form of the outstanding EPOCH study, a cluster multi-European centre RCT including 140000 children in which the bedside PEWS was shown to have no effect on reducing mortality in the intervention limb children. There was though, a difference in time to detection of deterioration and the focus has moved to this area in tool development. We should, therefore applaud, the initiative by the RCPCH, NHS England and NHS improvement described by Damian Roland and Simon Kenny to standardise the system, derive and use only a single score. The advantages are obvious: consistency; simplification of communicating trends between observers and hospitals to transcription errors possible when several scores are in circulation. There may not be an immediate reduction in mortality, but the advantages in everyone speaking the same language are clear. See page 648

\section{FETAL ALCOHOL SYNDROME}

Here's a paradox: for an issue as pervasive as fetal alcohol exposure and a phenotype as common as FAS, we know very little indeed about the epidemiology.
First recognised in the early 1970s when the classic (philtrum, upturned nose, epicanthus, palpebral fissure combination) phenotype was described. Prevalence estimates are complicated by the small number (likely less than 10\%) of children showing these signs, the rest of the iceberg manifesting much less specific neurobehavioural signs. Add to this the sensitivities around exposure information, making a social services decision based on uncertain data, issues around screening antenatally (there are biomarkers available) and the low yield in genetic work up series and the ways forward, other than primary prevention, become muddied. Read both Raja Mukerjhee's review and Zena Lam's series and make your own minds up whether FAS should fall into the (until recently) neglected disease bracket. See pages 653 and 636

\section{FEVER HOSPITALS}

We all know about the cyclical nature of history, but the timing of Philip Mortimer's 'Voices' paper about the London fever hospitals is uncannily good with respect to recent events and policy indecisiveness. The underpinning philosophy behind the hospitals was admirable: in Victorian England, beyond a degree of responsibility from poor law unions, there was effectively no central accountability for provision of care for febrile children from families of limited means. This era was the heyday of, among others, typhoid, scarlet fever, diphtheria and smallpox. With no viable alternatives, in 1867, Parliament took hold of the issue by the great philanthropophic leap of creating the 'Medical Asylum Board' whose main remit became the establishment of specific fever centres. After several decades in well-deserved limelight, the hospitals fell out of favour as much with parents as policy makers, the result of a combination of a change in infectious disease epidemiology, the recognition of the psychological harm to children that the prolonged spells in isolation could have and a creeping malaise around the risk of intra-hospital exposure. Darwin, aboard the Beagle, would no doubt have smiled wryly... See page 724

\section{ORCID iD}

Nick Brown http://orcid.org/0000-0003-1789-0436 